\title{
Evaluación del comportamiento productivo de tres variedades de papa sometidas a tres tipos diferentes de fertilización
}

Lorenzo Ante

Mario Montenegro

Carrera de Ingeniería Agropecuaria, Universidad Politécnica Salesiana, Guasaganda

\section{Introducción}

La evaluación de productividad de tres variedades de papa (dos mejoradas y una nativa) se realizó con la aplicación a un suelo que es franco arenoso de tres tipos de fertilizantes (orgánico; químico, y orgánico más químico). El estudio tuvo lugar en la Comunidad Guayama (parroquia Chugchilán, Municipio Sigchos, provincia de Cotopaxi) que está a $3.220 \mathrm{msnm}$, tiene una temperatura anual promedio de $12^{\circ} \mathrm{C}$ y registra una precipitación anual promedio de $900 \mathrm{~mm}$.

El patrón de cultivos de la zona no presenta mayores alternativas, es así que en la rotación de la siembra solo participan tres especies principales. El ciclo de barbecho es de uno a tres años. El ciclo agrícola, después del barbecho, está representado por una siembra de papas con variedades nativas como: coneja, chaucha, leona negra y piragua, y variedades mejoradas como: chilena, holandesa y castilla; luego haba, y finalmente cebada. Las variedades de papa son susceptibles a plagas y enfermedades, presentan cosechas tardías y tienen baja producción, todo lo cual ocasiona que no abastescan el consumo familiar.

La falta de asistencia técnica a los pequeños y medianos agricultores de la zona, quienes manejan los cultivos de forma tradicional, con cosechas poco productivas y no rentables; ha pro-

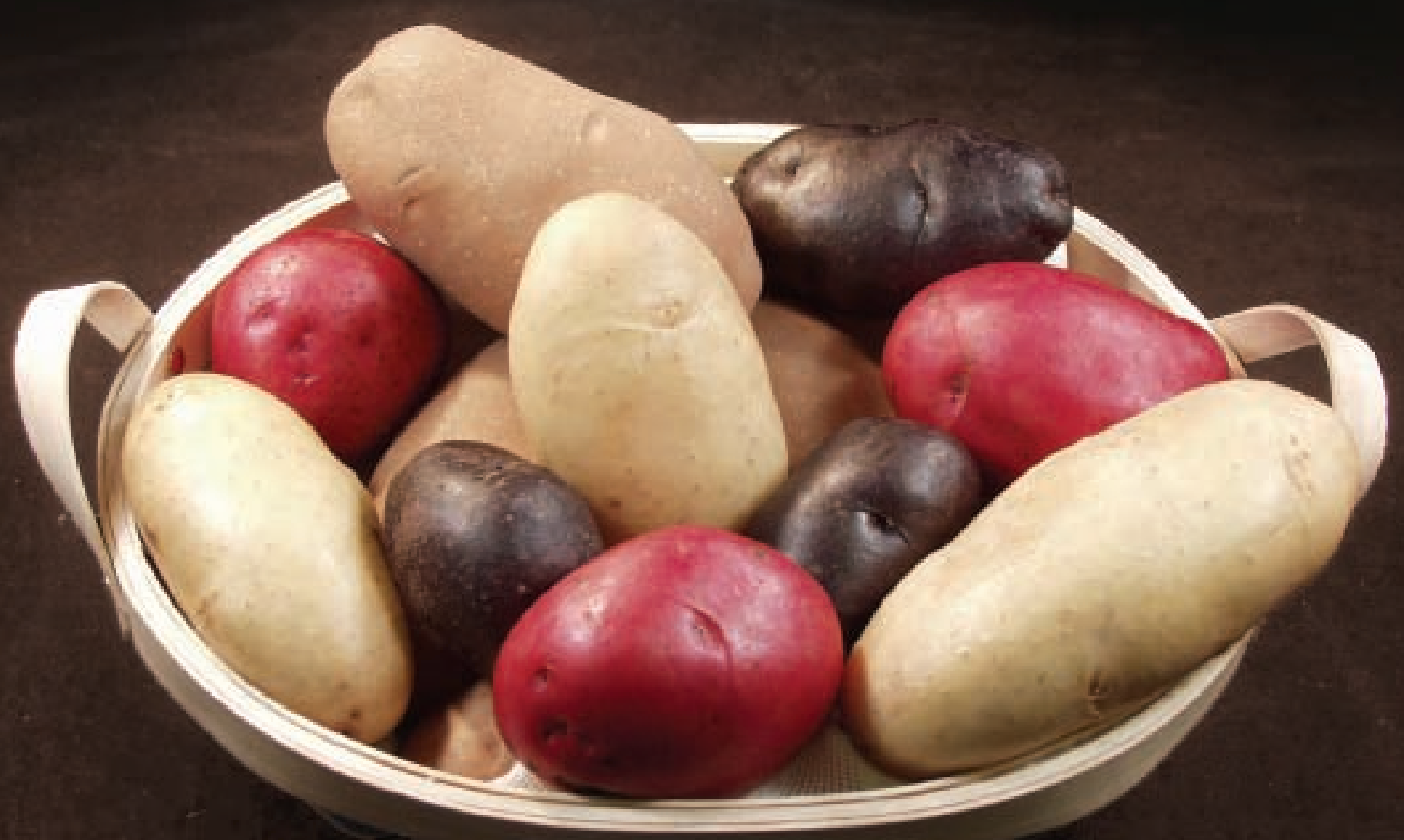


vocado que muchos miembros de una familia migren para tratar de completar el ingreso económico familiar. Es así, que se espera con este estudio poder contribuir a mejorar la subsistencia familiar, porque al aplicar una tecnología de manejo integrado en los cultivos de papas se puede mejorar la producción, sanidad y calidad.

Dentro de este escenario, el objetivo principal de esta investigación fue aplicar tecnologías adaptativas a los cultivos de papas, principalmente para la fertilización y mejora del rendimiento, así como transmitir los resultados a las comunidades locales.

\section{Métodos de investigación}

1. Área del experimento: Las superficies de suelo requeridas para el ensayo fueron:

a. Área total: $887,40 \mathrm{~m}^{2}(52,2 \times 17 \mathrm{~m})$

b. Área neta: $648,00 \mathrm{~m}^{2}$

c. Área útil: $24 \mathrm{~m}^{2} /$ parcela del suelo), Vitavax R 300 (desinfectante de semillas), Monitor 600 (insecticida) y Cymoxamil + Mancoseb (fungicida).

3. Análisis estadístico: Los factores en estudio considerados fueron las tres variedades de papas y los tres fertilizantes que se aplicaron a cada variedad, dando un total de nueve tratamientos $\left(\mathrm{V}_{1}\right.$ vs. $\mathrm{F}_{1}, \mathrm{~F}_{2}, \mathrm{~F}_{3} ; \mathrm{V}_{2}$ vs. $\mathrm{F}_{1}, \mathrm{~F}_{2}, \mathrm{~F}_{3} ; \mathrm{V}_{3}$ vs. $\mathrm{F}_{1}, \mathrm{~F}_{2}, \mathrm{~F}_{3}$. La evaluación del rendimiento se realizó con un diseño de bloques al azar (DBA), donde el arreglo factorial tuvo como factores las tres variedades de papa y como variables los tres tipos de fertilizantes distribuidos al azar en tres repeticiones que dieron un total de 26 tratamientos representados en las parcelas experimentales (Tabla 1).

5. Experimento: Los procedimientos y operaciones para implementar el experimento en el campo fueron:

Tabla 1. Esquema del análisis de varianza.

\begin{tabular}{lc}
\hline Fuentes de variación & Grados de libertad \\
\hline Repeticiones & 2 \\
Variedades & 2 \\
Fertilizantes & 2 \\
Variedades $v$ s. fertilizante & 4 \\
Error & 16 \\
TOTAL & 26 \\
\hline
\end{tabular}

La distancia de siembra fue: entre surcos $0,80 \mathrm{~m}$, entre cada planta 0,30 $\mathrm{m}$ y dos semillas/sitio.

2. Materiales: El experimento usó tres variedades $(\mathrm{V})$ de papas que fueron: Semichola $\left(\mathrm{V}_{1}\right.$ : mejorada), Esperanza $\left(\mathrm{V}_{2}\right.$ : mejorada) y Moroponcho $\left(\mathrm{V}_{3}\right.$ : nativa). Los fertilizantes $(\mathrm{F})$ aplicados fueron: abono orgánico ( $\mathrm{F}_{1}$ : excremento de cuyes y conejos), químico $\left(\mathrm{F}_{2}\right.$ : 10-30-10) y orgánicoquímico $\left(\mathrm{F}_{3}\right)$. Los agroquímicos usados fueron: Terraclor (desinfectante a. Preparación del suelo: Arada y rastreada con empleo de maquinaria agrícola; luego, surcada en forma manual con uso de azadón.

b. Siembra: Una vez desinfectados el suelo con Terraclor y la semilla con Vitavax, en dosis recomendadas por la casa comercial, se procedió a la siembra de dos tubérculos por sitio a distancias de $80 \times 20 \mathrm{~cm}$.

c. Fertilización: Se aplicaron tres quintales de abono orgánico (excremento de cuyes y conejos) a los trata- mientos 1, 4 y 7. Se aplicó una dosis de $40 \mathrm{lb}$ de fertilizante químico (1030-10) a los tratamientos 2,5 y 8 . Se aplicó una dosis del fertilizante orgánico-químico, en proporción de 1,5 qq - $20 \mathrm{lb}$, a los tratamientos 3, 6 y 9.

La fertilización se realizó durante la siembra, al fondo del surco y a chorro continuo, tapando después de la aplicación con una delgada capa de tierra.

6. Labores culturales: Se aplicaron los mismos procedimientos que realiza el agricultor papero, pero con otras oportunidades para el cultivo que fueron:

a. Rascadillo: Se efectuó a los 40 días después de la siembra para eliminar malezas.

b. Medio aporque: Se realizó a 30 días después del rascadillo para controlar malezas, aflojar el suelo, evitar pérdidas de humedad y brindar sostén a la planta.

c. Aporque: Se ejecutó a los 25 días de la labor anterior para cubrir los estolones y dar mejor sostén a las plantas.

7. Cosecha: Se realizó cuando los tubérculos alcanzaron la madurez fisiológica, es decir, cuando ellos se pueden pelar al frotar con los dedos.

Los tubérculos de cada tratamiento una vez cosechados fueron pesados, seleccionados y clasificados en seis categorías establecidas por los agricultores: Guanscha, Gruesa, Redroja, Redrojilla, Fina y Cuchi.

\section{Resultados}

El ensayo de papas sembrado en abril de 2005 en la Comunidad Guayama a $3.220 \mathrm{msnm}$, sufrió una sequía prolongada de aproximadamente dos meses durante su ciclo de cultivo, razón por la cual no se obtuvieron los resultados esperados.

En la Tabla 2 se presentan los datos de campo para rendimiento de cada variedad, fertilización e interacción de variedades $v s$. fertilizante.

Las respuestas que tuvo cada variedad de acuerdo al tipo de fertili- 
zante o su combinación, se calculó a través del análisis de varianza, cuyos resultados se presentan en la Tabla 3. Se observa que los rendimientos promedios entre variedades difieren en forma altamente significativa, la misma situación se produce con los fertilizantes; sin embargo, para la combinación variedades $\mathrm{x}$ fertilizantes las diferencias de rendimientos promedios alcanzan un nivel significativo de acuerdo a la Prueba de F.

El coeficiente de variación en este experimento es aceptable con un $8,6 \%$, pues depende en cierta forma de la disposición experimental, así como de la mayor o menor heterogeneidad del suelo (F para repeticiones no significativa es de 0,03 ). La diferencia del comportamiento de las variedades de papa respecto al rendimiento y la influencia de las fertilizaciones sobre

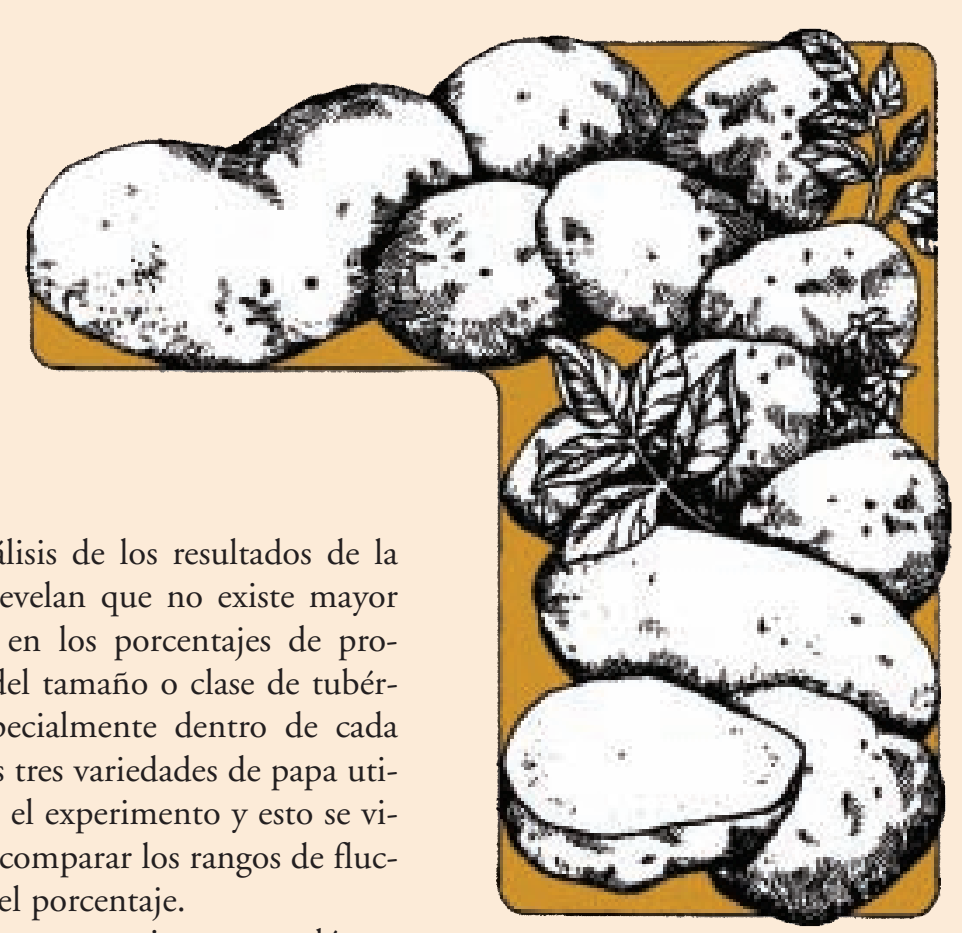
Tabla 5 revelan que no existe mayor variación en los porcentajes de producción del tamaño o clase de tubérculos, especialmente dentro de cada una de las tres variedades de papa utilizadas en el experimento y esto se visualiza al comparar los rangos de fluctuación del porcentaje.

El presente experimento reveló por un lado, que el mayor porcentaje de la producción de papa está en los grupos

Tabla 2. Datos de rendimiento en libras para cada una de las tres variedades de papa y de acuerdo a cada tratamiento aplicado.

\begin{tabular}{|c|c|c|c|c|c|c|c|c|c|c|}
\hline \multirow[t]{2}{*}{ Repeticiones } & \multicolumn{3}{|c|}{ Semilla } & \multicolumn{3}{|c|}{ Esperanza } & \multicolumn{3}{|c|}{ Moroponcho } & \multirow[t]{2}{*}{ TOTAL } \\
\hline & $\mathrm{O}$ & Q & $\mathrm{O}+\mathrm{Q}$ & $\mathrm{O}$ & Q & $\mathrm{O}+\mathrm{Q}$ & $\mathrm{O}$ & Q & $\mathrm{O}+\mathrm{Q}$ & \\
\hline \multirow{5}{*}{$\begin{array}{l}\text { I } \\
\text { I } \\
\text { III } \\
\text { Combinación V vs. F } \\
\text { Variedad }\end{array}$} & 24 & 86 & 73 & 52 & 88 & 85 & 26 & 71 & 44 & 548 \\
\hline & 24 & 82 & 72 & 58 & 92 & 73 & 25 & 73 & 46 & 543 \\
\hline & 25 & 104 & 70 & 51 & 89 & 71 & 26 & 72 & 42 & 549 \\
\hline & 73 & 272 & 215 & 161 & 269 & 229 & 76 & 215 & 131 & 1.640 \\
\hline & & 556 & & & 659 & & & 422 & & 1.640 \\
\hline Fertilizante & \multicolumn{3}{|c|}{ Orgánico 310} & \multicolumn{3}{|c|}{ Químico 756} & \multicolumn{3}{|c|}{$O+Q 575$} & 1.640 \\
\hline
\end{tabular}

$\mathrm{O}$ = orgánico; $\mathrm{Q}$ = químico; $\mathrm{V}$ = variedad; $\mathrm{F}$ = fertilizante.

un mismo carácter, se comprobó con la aplicación de la prueba de rango múltiple de Duncan (Tabla 4).

Después de la cosecha, se procedió a seleccionar los tubérculos dentro de cada una de las variedades de papa probadas en las seis categorías comúnmente utilizadas por los agricultores paperos y luego se realizó la comercialización. Así, en la Tabla 5 se presentan los datos de las variedades de papa por clase o tamaño del tubérculo.
Guansha y Gruesa (155 y 140 g/tubérculo) con un $64 \%$ de la producción varietal y por otro lado, que las clases de papa Redroja y Redrojilla, generalmente utilizadas por los pequeños agricultores para semilla, representan el $14 \%$.

La diferenciación más objetiva de los rendimientos obtenidos por los factores evaluados, variedades de papa y fertilizantes, así como sus combinaciones, se presentan transformados de libras/parcela a su equivalente en qq/ha.

\section{Variedades}

Las variedades de papa mejoradas de mayor producción promedio fueron Esperanza que alcanzó 229 qq/ha, seguida por Semichola con $187 \mathrm{qq} / \mathrm{ha}$, y en tercer lugar estuvo la variedad nativa Moroponcho con $141 \mathrm{qq} / \mathrm{ha}$ que fue utilizada como testigo.

Las tres variedades de papa mostraron diferencias con relación al ciclo vegetativo, siendo Esperanza un tanto precoz con 152 días de siembra a co- 
Tabla 3. Análisis de varianza para rendimiento promedio de Guayama (2005).

\begin{tabular}{lccccc}
\hline $\begin{array}{l}\text { Fuente de } \\
\text { Variación }\end{array}$ & $\begin{array}{c}\text { Grados de } \\
\text { Libertad }\end{array}$ & $\begin{array}{c}\text { Suma de } \\
\text { Cuadrados }\end{array}$ & $\begin{array}{c}\text { Cuadrados } \\
\text { Medios }\end{array}$ & F & Significancia \\
\hline Total & 26 & $15.862,17$ & - & - & NS \\
Repeticiones & 2 & 2,05 & 1,03 & 0,03 & $* *$ \\
Variedades & 2 & $3.148,67$ & $1.574,33$ & 56,57 & $* *$ \\
Fertilizantes & 2 & $1.181,55$ & 590,78 & 21,23 & $*$ \\
Variedad & 4 & $1.084,61$ & 271,15 & 9,74 & \\
vs. fertilizante & 16 & 445,29 & 27,83 & & \\
Error & & & & \\
\hline
\end{tabular}

Tabla 4. Prueba de rango múltiple de Duncan para diferenciar el comportamiento de las variedades de papa respecto al rendimiento y la influencia de las fertilizaciones sobre un mismo carácter.

\begin{tabular}{lll}
\hline Variedad & Promedio (lb) & Rango \\
Esperanza & 73,16 & A \\
Semichola & 62,16 & B \\
Moroponcho (T) & 41,83 & B \\
& & Rango \\
Fertilizante & Promedio (lb) & A \\
Químico & 83,94 & B \\
Orgánico + Químico & 63,83 & B \\
Orgánico & 62,16 & \\
& & Rango \\
Variedades vs. fertilizante & Promedio (lb) & A \\
Semichola vs. Químico & 90,67 & A \\
Esperanza vs. Químico & 89,67 & B \\
Esperanza vs. Orgánico + Químico & 76,33 & B \\
Moroponcho vs. Químico & 71,67 & B \\
Semichola vs. Orgánico + Químico & 71,66 & $\mathrm{C}$ \\
Esperanza vs. Orgánico & 53,67 & $\mathrm{C}$ \\
Moroponcho vs. Orgánico & 43,67 & $\mathrm{D}$ \\
Moroponcho vs. Orgánico & 25,33 & $\mathrm{D}$ \\
Semichola vs. Orgánico & 24,33 & \\
\hline
\end{tabular}

La misma letra indica que no hay diferencia estadística en el rango.

secha; Semichola con 162 días, y Moroponcho con 181 días; resaltándose, que este es un factor importante y debe ser considerado durante la siembra para disminuir los riesgos con relación al clima.

\section{Fertilizantes}

La respuesta del suelo a la aplicación de los fertilizantes orgánico, químico y orgánico-químico se observó en la producción, así de acuerdo a la fertilización, el primer lugar obtuvo el fertilizante químico con $350 \mathrm{qq} / \mathrm{ha}$, el segundo el orgánico-químico con 266 qq/ha y el tercero el orgánico con $144 \mathrm{qq} / \mathrm{ha}$.

Es bastante notable la diferencia de rendimiento que existe entre la fertilización química y la orgánica (206 qq/ha), así como entre la fertilización orgánico-química vs. la orgánica (122 qq/ha). En este sentido, se aclara que por el tipo de experimento se esperaba que los rendimientos de papa sean inferiores con el abono orgánico y el químico-orgánico, porque su efecto es residual, lo cual en la misma siembra contrastó con la respuesta que hubo con el fertilizante inorgánico.

Interacción fertilizante vs. variedad

La comparación de las respuestas obtenidas para rendimiento por hectárea (qq/ha) de los tres tipos de fertilización combinada con las tres variedades dio como resultado que la mejor es la química, especialmente cuando 
Tabla 5. Clasificación de los tubérculos de acuerdo al peso en gramos (g) y el porcentaje (\%) por variedad.

\begin{tabular}{l|cc|cc|cc}
\hline \multirow{2}{*}{ Clasificación } & \multicolumn{7}{c}{ Variedad } \\
\hline & \multicolumn{2}{|c|}{ Semichola } & \multicolumn{2}{c}{ Esperanza } & \multicolumn{2}{c}{ Moroponcho (Test) } \\
\cline { 2 - 7 } & $\mathrm{g}$ & $\%$ & $\mathrm{~g}$ & $\%$ & $\mathrm{~g}$ & $\%$ \\
Guanscha & 155 & 33.76 & 165 & 32.35 & 150 & 72.22 \\
Gruesa & 140 & 30.50 & 150 & 29.41 & 120 & 29.77 \\
Redroja & 80 & 17.42 & 95 & 18.62 & 65 & 16.12 \\
Redrojilla & 50 & 10.89 & 60 & 11.76 & 40 & 9.92 \\
Fina & 25 & 5.44 & 30 & 5.88 & 20 & 4.96 \\
Cuchu & 9 & 1.96 & 10 & 1.96 & 8 & 1.98 \\
\hline
\end{tabular}

es aplicada a la papa Semichola (378 qq/ha), Esperanza (374 qq) y Moroponcho (298 qq). Los resultados de rendimiento de siembra fueron menores con el fertilizante orgánico-químico aplicado a las variedades de papa Esperanza (317 qq), Semichola (299 qq) y Moroponcho (182 qq). El resultado más bajo de producción fue con el abono orgánico aplicado a las variedades de papa Esperanza (224 qq), Moroponcho (106 qq) y Semichola (101 qq/ha).

En general, el efecto de las combinaciones de variedades de papa $v$ s. el tipo de fertilización fueron positivas; sin embargo, el mejor rendimiento fue con la fertilización química. Aclarándose, que la variedad Semichola cuando es cultivada con abono orgánico tiene una respuesta inferior a la
Moroponcho, siendo esta última una variedad nativa, erosionada, de ciclo vegetativo tardío y con tasas de baja producción.

\section{Consideraciones finales}

La presente investigación tuvo como principal inconveniente no controlado un período de sequía, situación que incidió en que los resultados obtenidos no sean los esperados.

El comportamiento de las variedades de papas sometidas a los tres tipos de fertilizantes denotó que existe un ciclo vegetativo varietal diferenciado, pudiéndose catalogar a la variedad Esperanza como precoz con cinco meses y considerando que fue sembrada a 3.200 msnm presentó un mayor rendimiento por unidad de superficie.
La mayor producción de cultivos de papa se obtuvo con la fertilización química y la menor fue con la orgánica.

Por lo que respecta a la interacción variedad vs. fertilizante existieron repuestas similares en rendimiento para Semichola y Esperanza, particularmente en combinación con la fertilización química.

Los resultados de este experimento indican que: la mejor época para la siembra es la entrada del período de lluvias; el abono orgánico debe ser aplicado en todos los cultivos para mejorar los suelos; el análisis del suelo permite aplicar la dosis recomendada de fertilizante químico y materia orgánica en el cultivo, y que se debe procurar sembrar variedades mejoradas en la zona como son Esperanza y/o Semichola. 